\title{
TRANSFERENCIA DE CALOR E POTÊNCIA CONSUMIDA NA AGITAÇÃO DE CARBOXIMETILCELULOSE EM UM TANQUE COM IMPULSOR MECÂNICO E SERPENTINA EM ESPIRAL
}

\author{
L. G. C. GUIMARÃES ${ }^{1}$, B. S. CRUZ ${ }^{1}$, M. E. G. MANTYK ${ }^{1}$, \\ V. S. ROSA ${ }^{1,2}$ e D. MORAES JÚNIOR ${ }^{1}$ \\ ${ }^{1}$ Universidade Santa Cecília, Departamento de Engenharia Química \\ ${ }^{2}$ Universidade de São Paulo, Escola Politécnica, Departamento de Engenharia Química \\ E-mail para contato: le_gcg@hotmail.com
}

\begin{abstract}
RESUMO - Os tanques com impulsores mecânicos são largamente utilizados em processos como reatores químicos e trocadores de calor. As serpentinas em espiral podem ser empregadas para o aquecimento devido ao seu baixo custo. $\mathrm{O}$ seu projeto envolve a determinação do número de Nusselt e da potência consumida, entretanto, quando envolve fluidos não-newtonianos, a literatura apresenta poucas informações disponíveis. O presente estudo visou analisar o número de Nusselt e o número de potência no aquecimento de solução aquosa de carboximetilcelulose (CMC) em um tanque equipado com uma serpentina em espiral e dois impulsores mecânicos. A unidade experimental consistiu de um tanque com diâmetro de $400 \mathrm{~mm}$, um motor elétrico de $2 \mathrm{hp}, 1$ impulsor axial com 4 pás inclinadas a $45^{\circ}, 1$ impulsor radial turbina e 1 serpentina em espiral de cobre. Foram realizados 5 ensaios com cada impulsor mecânico, variando-se a rotação, medindo-se a temperatura em 3 pontos específicos no tanque a cada 1 minuto. Também mensurou-se a potência consumida em cada rotação através da medição do torque com o motor em balanço sobre rolamentos. Com os dados obtidos foram calculados os valores do número de Nusselt, Reynolds e número de potência e plotaram-se os mesmos. Pode-se observar que o impulsor axial fornece o menor consumo de energia e que para Reynolds inferiores a 400 (cerca de $300 \mathrm{rpm}$ ), o mesmo possui um maior valor de Nusselt, tornando-se assim o recomendado para agitação e aquecimento em sistemas semelhantes ao proposto no trabalho.
\end{abstract}

\section{INTRODUÇÃO}

As serpentinas em espiral são empregadas no aquecimento e resfriamento de líquidos em tanques com agitação devido ao seu baixo custo. Essas unidades podem ser usadas em uma grande variedade de processos, como na produção de tintas, produtos alimentícios, produtos farmacêuticos, polímeros e combustíveis. O projeto da área (A) de uma serpentina em espiral é uma função do coeficiente global de transmissão de calor $U$, da carga térmica $(\mathrm{Q})$ e da diferença média de temperaturas entre os fluidos quente e frio (LMTD), como apresentado na Equação 1. 


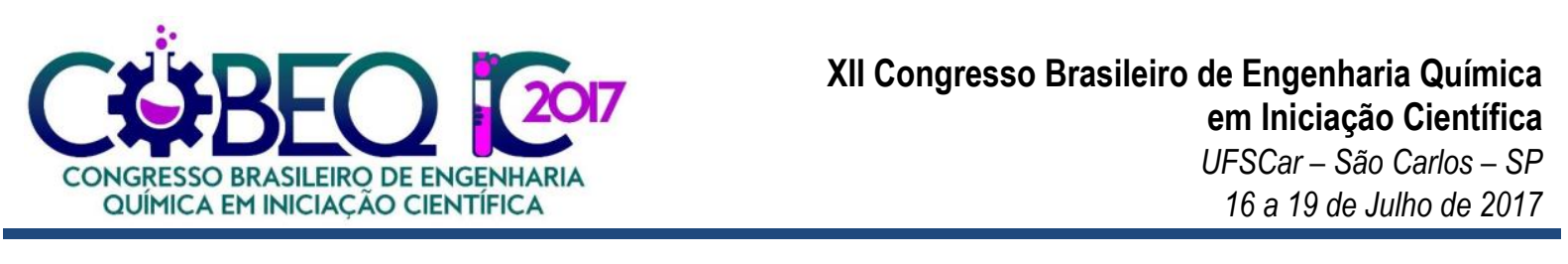

$$
A=Q / U \cdot L M T D
$$

No projeto da área de troca térmica com fluidos não-newtonianos, como as soluções aquosas de polímeros (carboximetilcelulose, carbopol 940), é necessário a determinação de um modelo reológico que explique a variação da viscosidade aparente do fluido agitado em agitação (Chhabra e Richardson, 2011). Um modelo largamente utilizado para fluidos independentes do tempo é o descrito pela lei das potências (Equação 2), o qual relaciona a viscosidade aparente $(\eta)$ com a taxa de cisalhamento $(\operatorname{grad} \vec{v})$ através de duas constantes empíricas, o fator de consistência (k') e o índice de consistência (n), obtidas experimentalmente.

$$
\eta=k^{\prime} \operatorname{grad} \vec{v}^{n}
$$

A determinação do coeficiente $U$, em soluções poliméricas, é obtida como uma função do número de Nusselt $(\mathrm{Nu})$, o qual depende da variação dos números de Reynolds ( $\mathrm{Re}$ ) e Prandtl (Pr) e da relação entre a viscosidade do fluido em agitação na temperatura "bulk" e da viscosidade do fluido na temperatura da superfície externa do tubo da serpentina em espiral. Ressaltando que os números adimensionais e a relação supracitada devem ter o modelo reológico nas suas equações. A potência consumida é um parâmetro de grande importância pois quantifica o consumo energético do impulsor mecânico ao promover a agitação. Dependendo do tipo de impulsor utilizado (axial ou radial) o consumo de energia pode ter uma grande variação, porém, a troca térmica pode permanecer inalterada. Tradicionalmente, a potência consumida é quantificada através do número de potência, o qual é função do número de Reynolds, do tipo de impulsor, da geometria do tanque e, principalmente, da reologia do fluido Em relação a serpentina em espiral observou-se que há poucas informações sobre a análise da transmissão de calor e do consumo de potência nessas unidades durante a agitação e aquecimento de fluidos não-newtonianos, em sistemas operando em batelada e de forma contínua. $\mathrm{O}$ presente estudo visou determinar a relação entre o número de Nusselt e o número de potência em função do número de Reynolds, no aquecimento de uma solução aquosa de carboximetilcelulose com concentração de $1,5 \%$ em um tanque equipado com uma serpentina espiral, em função de um impulsor axial com 4 pás inclinadas e um impulsor radial tipo turbina com 6 pás planas.

\section{MATERIAL E MÉTODO}

\subsection{Procedimento experimental}

A Figura 1 apresenta um esquema da unidade experimental, localizada no Laboratório de Operações Unitárias da Universidade Santa Cecília, em Santos - SP, com os seus periféricos de forma detalhada. Foi utilizado como fluido quente, o qual foi colocado no interior da serpentina em espiral, água destilada com temperatura e vazão volumétrica de entrada constantes, em $65^{\circ} \mathrm{C}$ e 1 litro por minuto, respectivamente. Como fluido frio, foi utilizada uma solução aquosa de carboximetilcelulose (CMC) com concentração mássica de 1,5\%, a qual foi alocada no interior do tanque. O processo foi conduzido em batelada. Foram colocados três termopares em posições distintas no interior do tanque, visando obter a variação de temperatura da solução de CMC, e, um termopar na entrada da serpentina em espiral e outro na saída. 
Figura 1 - Fluxograma da unidade experimental - (1) Banho ultratermostatizado; (2) Bomba para o transporte do fluido quente; (3) Válvulas de esfera; (4) Rotâmetro; (5) Termopar de medição da temperatura de entrada do fluido quente; (6) Serpentina em espiral; (7), (10) e (11) Termopares de medição da temperatura de aquecimento do fluido frio; (8) Tanque de agitação com volume de 50 litros; (9) Isolante térmico; (12) Impulsor mecânico; (13) Motor elétrico sobre rolamentos; (14) Termopar de medição da temperatura de saída do fluido quente; (15) Trocador de calor.

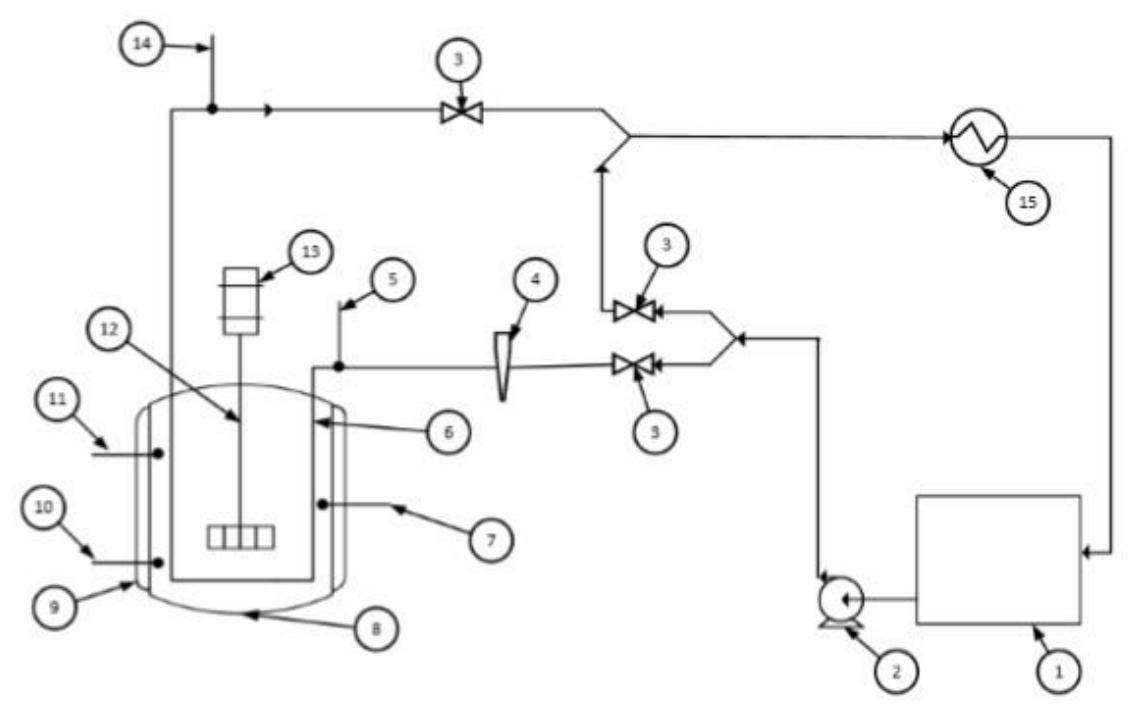

O estudo foi dividido em duas etapas: análise da transferência de calor e consumo de potência pelos impulsores mecânicos. Em relação à transmissão de calor, foram realizados cinco ensaios de aquecimento para cada um dos impulsores mecânicos, totalizando 10 ensaios, sendo que o parâmetro independente era a rotação, a qual foi variada em 100, 300, 500, 700 e $900 \mathrm{rpm}$. Como resposta, foram medidas as temperaturas mensuradas pelos 5 termopares supracitados em um intervalo de tempo de 1 minuto, sendo que o final do ensaio ocorria quando a diferença de temperatura entre o início e o final do aquecimento da solução de CMC era de pelo menos $15^{\circ} \mathrm{C}$. Os ensaios referentes ao consumo de potência foram realizados colocandose o motor elétrico dos impulsores mecânicos em balanço sobre rolamentos, visando medir a força $(\mathrm{F})$ gerada para cada rotação $(\mathrm{N})$ em função do braço acoplado (B), dessa forma, a potência consumida é calculada conforme apresentado na Equação 3. Nesses ensaios variou-se a rotação de 100 a $900 \mathrm{rpm}$.

$$
P=2 \pi N B F
$$

As análises reológicas foram realizadas em um reômetro DV-III Brookfield localizado no laboratório de Separações Térmicas e Mecânicas da Escola Politécnica da Universidade de São Paulo.

\subsection{Fundamentação teórica}

Transferência de calor: Inicialmente foi realizado um balanço de energia na unidade experimental (contemplando apenas o tanque de agitação e a serpentina em espiral conforme apresentado na Figura 2) descrito pela $1^{a}$ lei da termodinâmica (Equação 4), considerando as 
seguintes hipóteses: a) tanque perfeitamente agitado, b) perda de calor ao ambiente externo desprezível, devido ao isolamento térmico do tanque, c) trabalho de eixo pelo impulsor mecânico desprezível e d) variação de energia cinética e potencial insignificantes em relação aos termos da entalpia.

$$
w_{h} c_{p h}\left(T_{1}-T\right)=M c_{p c} d t_{b} / d \theta
$$

Sendo M, a massa da solução de CMC colocada no tanque, $\theta$, o tempo do aquecimento, $\mathrm{C}_{\mathrm{pc}}$, o calor específico da solução de $\mathrm{CMC}, \mathrm{C}_{\mathrm{ph}}$, o calor específico do fluido quente, $\mathrm{w}_{\mathrm{h}}$, a vazão em massa do fluido quente, $\mathrm{T}_{1}$, a temperatura de entrada do fluido quente na serpentina, $\mathrm{T}, \mathrm{a}$ temperatura de saída do fluido quente da serpentina e $\mathrm{t}_{\mathrm{b}}$, a temperatura "bulk" da solução de CMC durante a agitação. O trabalho proposto por Rosa, et. al. (2017) aplica a Equação 4 em um sistema de agitação semelhante ao usado nesse estudo, porém em operação contínua, dessa forma, adaptando a este processo (o qual é em batelada), logo, a equação 4 pode ser escrita em função do coeficiente U e da área de troca térmica (A), como ilustrado na Equação 5.

$$
U=\frac{1}{A\left(T_{1}-T\right)}\left[\ln \left(T_{1}-t_{b} / T-t_{b}\right)\right]\left[M c_{p c} \frac{d t_{b}}{d \theta}\right]
$$

A solução da Equação 5 é obtida com uma integração fixando-se uma condição inicial para a temperatura "bulk" no tempo, entretanto, deve-se conhecer a variação do coeficiente U com o tempo, o que a princípio não é possível. Dessa forma, a Equação 5 deve ser resolvida através de uma discretização no tempo, calculando o valor do coeficiente $U$ em cada intervalo especificado de tempo e verificando se há ou não uma tendência de variação. Constatou-se que no presente estudo, o coeficiente $\mathrm{U}$ flutuou em torno de um valor médio ao decorrer do tempo, não apresentando uma tendência, dessa forma, conclui-se que para o aquecimento de soluções aquosas de carboximetilcelulose na concentração estudada, o coeficiente $U$ independe do tempo de ensaio. Logo, para os cálculos posteriores, foi calculado o valor médio do coeficiente $U$ em cada ensaio. A temperatura "bulk" pode ser calculada a partir da média de temperatura dos três termopares em cada instante de tempo, caso a variação de temperatura entre cada termopar seja irrelevante, o que ocorreu nesse estudo, fornecendo uma variação percentual de no máximo $1,5 \%$ em todos os ensaios. Dessa forma foi comprovado que nas condições estudadas, o tanque está na condição de perfeitamente agitado. Ressaltando, que todas as temperaturas que estão presentes na Equação 5 foram mensuradas ao longo do tempo, conforme descrito de forma detalhada na seção 2.1. Com os valores obtidos do coeficiente $U$, foi determinado o valor do coeficiente interno de convecção $\left(h_{i o}\right)$, pela equação de Gnielinski (1976) para cada ensaio, dessa forma, possibilitando o cálculo do coeficiente externo de convecção $\left(h_{0}\right)$ como apresentado na Equação 6 a e do número de Nusselt (Nu), na Equação 6 b.

$$
h_{o}=\frac{U h_{i o}}{h_{i o}-U} \quad(6 a) \quad N u=\frac{h_{o} D_{t}}{k}
$$

Em que $\mathrm{D}_{\mathrm{t}}$ é o diâmetro do tanque e $\mathrm{k}$ é a condutividade térmica do CMC. Foi verificado no reômetro que a solução de CMC utilizada no presente estudo possui um comportamento de um fluido independente do tempo do tipo pseudoplástico, dessa forma o uso da equação 2 (lei das potências) é permitido. Incorporando a equação 2 no número de Reynolds $(\operatorname{Re})$ e considerando a metodologia, descrita por Metzner e Otto (1957) da relação entre a rotação do impulsor mecânico e o gradiente de velocidades, o Reynolds modificado é calculado pela equação 7. 


$$
R e=\frac{\mathrm{N}^{2-\mathrm{n}} \mathrm{Da}^{2} \rho}{\mathrm{k}^{\prime} \mathrm{ks}^{\mathrm{n}-1}}
$$

Sendo $\mathrm{N}$ a rotação do impulsor mecânico, $\mathrm{D}_{\mathrm{a}} \mathrm{o}$ diâmetro do impulsor mecânico, $\rho$ a massa específica da solução de CMC e ks a constante de proporcionalidade entre o gradiente de velocidades e a rotação do impulsor mecânico, proposto por Metzner e Otto. Visando obter as constantes reológicas ( $k^{\prime}$ e $n$ ), do modelo da lei das potências, em função da temperatura "bulk", durante cada ensaio, foram coletadas três amostras, sendo uma na temperatura de início do ensaio, a segunda em uma temperatura intermediária e a terceira na temperatura final do ensaio, ressaltando que a variação de temperatura inicial e final da solução de CMC era de $15^{\circ} \mathrm{C}$. Cada amostra foi submetida ao reômetro, e para cada ensaio, obteve-se uma expressão para cada constante reológica em função da temperatura. Como o modelo da lei das potências descreve a variação da viscosidade aparente, a qual é uma propriedade, todas os valores de k' e n foram calculados na temperatura "bulk" média.

Potência consumida: Com os valores calculados da potência através da Equação 3, calculou-se o número de potência (Np), conforme apresentado na Equação 8.

$$
N p=P / \rho N^{3} D_{a}{ }^{5}
$$

\section{RESULTADOS}

Com os valores calculados do coeficiente $\mathrm{U}$, número de Nusselt, número de Reynolds e número de potência, foi possível realizar algumas considerações. A Figura 2 apresenta a variação do número de Nusselt com o número de Reynolds para os dois impulsores mecânicos, axial e radial.

Figura 2 - Variação do número de Nusselt com o número de Reynolds, observada para os impulsores axial e radial.

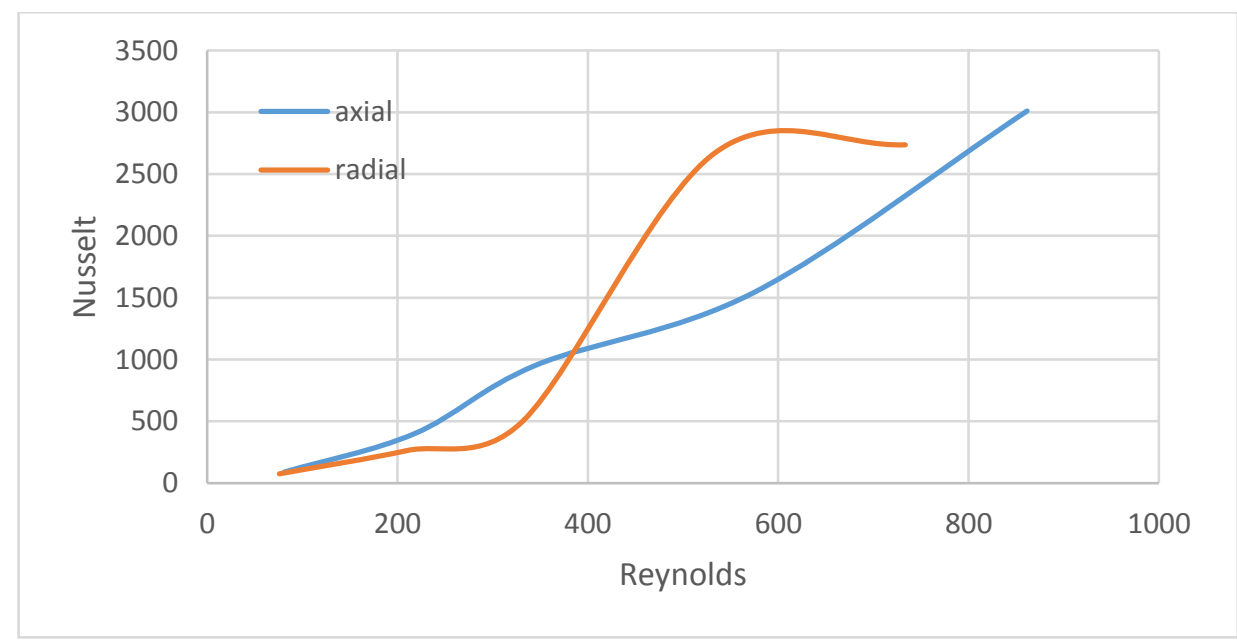

Na Figura 3 são apresentados os valores calculados do número de potência com o número de Reynolds para os impulsores axial e radial. 
Figura 3 - Variação do número de potência com o número de Reynolds, observada para os impulsores axial e radial.

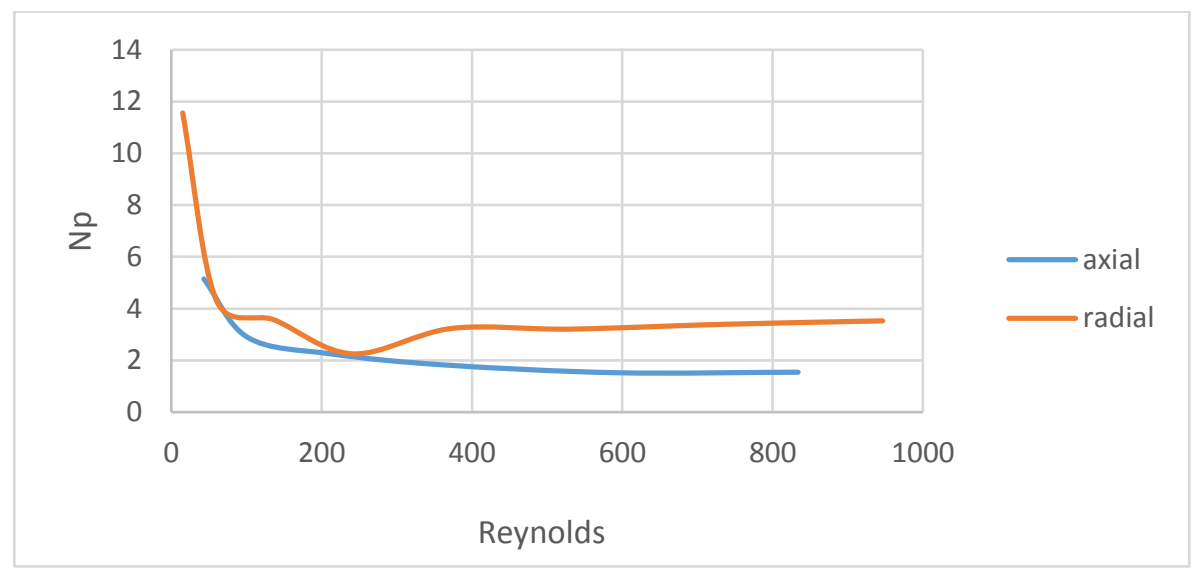

Observou-se, na Figura 2, que toda a faixa de número de Reynolds obtida foi na região laminar, pois todos os valores foram inferiores a 2000, o que já era esperado, devido à elevada consistência da solução de CMC. Com valores de Reynolds abaixo de 400 (cerca de 300 rpm), o aquecimento foi mais eficiente com o uso do impulsor axial, devido o mesmo ordenar o escoamento e direcionar o fluido à base do tanque, onde está localizada a serpentina em espiral. Entretanto, para Reynolds acima de 400, o impulsor radial passou a ser o mais eficiente, devido ao aumento da intensidade do escoamento, mesmo estando na região laminar. Na Figura 3, constatou-se que para toda a extensão dos valores de Reynolds, o número de potência foi maior com impulsor radial, quando comparado com impulsor axial. Isso ocorre, pois, o impulsor radial propulsiona o fluido em um escoamento tangencial ao eixo do impulsor, ou seja, é necessário um maior torque quando comparado ao impulsor axial, que direciona o fluido de forma ordenada ao fundo do tanque, tornando o impulsor axial o mais viável economicamente.

\section{CONCLUSÃO}

Conclui-se que, para o aquecimento da solução de CMC ou de outras soluções poliméricas que sigam o modelo reológico da lei das potências, o impulsor axial é o mais recomendado, devido na faixa de operação industrial (rotações de até $300 \mathrm{rpm}$ ) o mesmo fornece uma troca térmica superior ao impulsor radial e com um menor consumo de potência.

\section{REFERENCIAS}

CHHABRA, R. P.; RICHARDSON, J. F., Non-newtonian flow and applied rheology. Engineering applications. Oxford: Butterworth-Heinemann, 2011;

GNIELINSKI, V., Int. Chem. Eng., 16, 359, 1976;

METZNER, A. B.; OTTO, R. E. Agitation of non-Newtonian fluids. AIChE Journal, v. 3, n. 1, p. 3-10, 1957;

ROSA, V. S.; TAQUEDA. M. E. S.; PAIVA, J. L.; MORAES, M. S.; MORAES JÚNIOR, D., Nusselt's correlations in agitated tanks using the spiral coil with Rushton turbine and $\mathrm{PBT} 45^{\circ}$ impeller. Comparison with tanks containing vertical tube baffles. Appl. Therm. Eng., 110, p. 1331-1342, 2017; 